\title{
Random Linear Network Coding for Satellite-Aided Flight Data Streaming
}

\author{
Ioannis Chatzigeorgiou and Catherine Price \\ School of Computing and Communications, Lancaster University \\ Lancaster LA1 4WA, United Kingdom \\ Email: \{i.chatzigeorgiou, c.price\}@lancaster.ac.uk
}

\begin{abstract}
In response to the recommendation by the International Civil Aviation Organization for the enhancement of flight tracking, Inmarsat developed a platform that allows flight data recorder information to be streamed off aircrafts to defined recipients via its geostationary satellites. This paper considers various data forwarding mechanisms and demonstrates that random linear network coding can support reliable transmission that is more resilient to channel errors. Resilience can be further improved if partial packet recovery is enabled and if authorized users with receive-only capabilities share retrieved data with aviation safety recipients.
\end{abstract}

\section{INTRODUCTION}

Flight Data Recorders (FDRs), commonly known as black boxes, are fitted to commercial aircraft to record a range of parameters during a flight. In the event of an accident, the recorded information is recovered and analyzed in an effort to diagnose the cause of the accident. Problems emerge when the recorded data has been corrupted (e.g., flight RQ904) or the FDR cannot be retrieved because the wreckage of an aircraft cannot be reached or cannot be located (e.g., flight MH370).

Since the MH370 incident in 2014, proposals for live streaming flight data via geostationary satellites started to emerge [1]. The International Civil Aviation Organization (ICAO) proposed the concept of the Global Aeronautical Distress and Safety System (GADSS) to address requirements for global flight tracking, autonomous distress tracking and flight data recovery. According to ICAO, airlines are required to regularly transmit highly accurate position information from November 2018 and additional flight data from January 2021 [2]. Inmarsat, in collaboration with Cobham, Thales and Honeywell, developed the SwiftBroadband-Safety (SB-S) communication platform [3] as a means to support all GADSS requirements. The Transmission Control Protocol (TCP) has been adopted by SwiftBroadband services for data transmission [4]. In late 2017, the SB-S platform entered service evaluation on a commercial aircraft [5].

This paper considers TCP-like transmission, referred to as uncoded transmission, of flight data from a satellite to a main station, e.g., an aviation safety recipient, and investigates other forwarding schemes that can improve the resilience of the system. The motivation for this paper

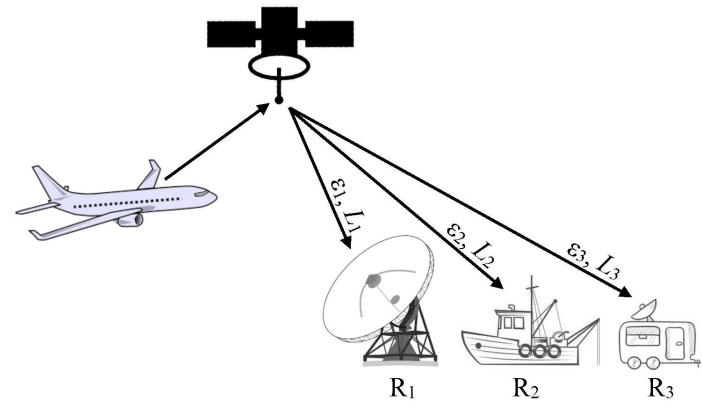

Fig. 1. Example of a system, in which a satellite relays flight data to three receivers over channels characterized by different packet erasure probabilities $\left(\varepsilon_{1}, \varepsilon_{2}, \varepsilon_{3}\right)$ and average burst lengths $\left(L_{1}, L_{2}, L_{3}\right)$.

is to gauge the benefits of index coding [6] and random linear network coding [7], without or with partial packet recovery [8], when main stations collaborate either with each other or with auxiliary stations, e.g., users that have been authorized to receive flight data.

The paper has been structured as follows: Section II presents the system model, the channel model and the considered system configurations. Section III describes the schemes under investigation that can be used by a satellite to relay flight information. Performance results are discussed in Section IV and key findings are summarized in Section V.

\section{System Model}

As shown in Fig. 1, we consider an aircraft transmitting packets that contain vital flight data to a satellite, which stores them on board and uses a forwarding scheme to relay them to authorized receivers $\mathrm{R}_{1}, \ldots, \mathrm{R}_{S}$. We classify receivers into main stations, which use feedback channels to request packet retransmissions from the satellite, and auxiliary stations, which can only be in receiving mode. If $K$ data packets have been collected and stored on the satellite, the relaying process is divided into two stages. In the broadcast stage, the $K$ data packets are forwarded to the receivers. In the retransmission stage, additional packets are generated and transmitted, based on the adopted forwarding scheme and feedback from main stations. We denote by $N$ the maximum allowable number of 
transmitted packets over the two stages, where $N \geq K$. Given that each additional packet in the retransmission stage requires energy from the satellite and introduces delay to the recovery of the flight data, achieving a value of $N$ as close as possible to $K$ will ensure that $K$ data packets' worth of information will be delivered at the lowest energy and delay cost.

The Gilbert channel [9] has been used in this paper to model packet losses, also referred to as packet erasures, between the satellite and a receiver. This simple model can capture the bursty nature of the satellite channel, i.e., the occurrence of consecutive packet erasures, and is often used in the literature when satellite communication schemes are assessed, e.g., [10] and [11]. The Gilbert channel is a two-state Markov chain, according to which packets are correctly received during a 'good' state but are erased during a 'bad' state. Transitions between the two states occur with probabilities $p_{g b}, p_{g g}, p_{b g}$ and $p_{b b}$, where $p_{g g}=1-p_{g b}$ and $p_{b b}=1-p_{b g}$, as depicted in Fig. 2. The steady-state probability of being in the 'bad' state represents the packet erasure probability and is given by $\varepsilon=p_{g b} /\left(p_{g b}+p_{b g}\right)$ [11], [12]. The expected number of consecutive packet erasures provides the average length of an erasure burst, and is equal to $L=1 / p_{b g}$ [11]. In this paper, parameters $\varepsilon$ and $L$ are taken to be given quantities. For this reason, the transition probabilities have been expressed in terms of $\varepsilon$ and $L$, i.e., $p_{b g}=1 / L$ and $p_{g b}=\varepsilon /[L(1-\varepsilon)]$. Index $i$ has been appended to $\varepsilon$ and $L$ as a reference to the packet erasure probability and the average burst length, respectively, of the link between the satellite and receiver $\mathrm{R}_{i}$, as shown in Fig. 1 .

The following three configurations have been considered, based on the type and connectivity of the receivers:

- Independent main stations: Each main station transmits feedback messages to the satellite in an effort to collect sufficient packets and recover the flight data.

- Connected main stations: The main stations act independently during both stages. If the packets transmitted by the satellite reach the maximum allowable value and one or more stations have not recovered the flight data, the stations use a reliable backbone network to exchange packets in real time or offline.

- Main station connected to auxiliary stations: A single main station requests packets through a feedback channel in the retransmission stage, while auxiliary stations are in receiving mode in both stages. If the main station is not successful in recovering the flight data by the end of the retransmission stage, it uses reliable terrestrial links to collect any missing packets from auxiliary stations in real time or offline.

This section provided details about the system setup, the channel model and the underlying assumptions. The candidate schemes for forwarding packets in the retransmission stage are discussed in the following section.

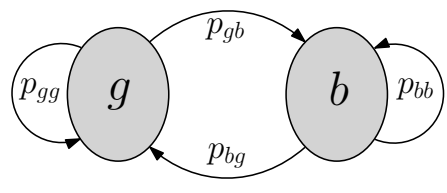

Fig. 2. The Gilbert channel model, where $g$ and $b$ represent the 'good' state and the 'bad' state, respectively.

\section{FORWARDING SCHEMES}

Let $\mathbf{U}=\left[\begin{array}{lll}\mathbf{u}_{1} \ldots \mathbf{u}_{K}\end{array}\right]^{\mathrm{T}}$ be a matrix composed of $K$ packets of flight data that have been successfully received by the satellite. The packets that will be transmitted by the satellite during the two stages can be expressed as follows:

$$
\mathbf{X}=\mathbf{G} \mathbf{U} \Leftrightarrow\left[\begin{array}{c}
\mathbf{x}_{1} \\
\vdots \\
\mathbf{x}_{K+\Delta}
\end{array}\right]=\left[\frac{\mathbf{I}_{K \times K}}{\mathbf{C}_{\Delta \times K}}\right]\left[\begin{array}{c}
\mathbf{u}_{1} \\
\vdots \\
\mathbf{u}_{K}
\end{array}\right] .
$$

Matrix $\mathbf{G}$ is the vertical concatenation of the $K \times K$ identity matrix $\mathbf{I}_{K \times K}$ and the $\Delta \times K$ matrix $\mathbf{C}_{\Delta \times K}$, where $\Delta \leq N-K$. Note that $\mathbf{I}_{K \times K}$ represents the broadcast stage and $\mathbf{C}_{\Delta \times K}$ characterizes the forwarding scheme that is used in the retransmission stage. The column vector $\mathbf{x}$ contains the transmitted packets $\mathbf{x}_{1}, \ldots, \mathbf{x}_{K+\Delta}$.

At the end of the two-stage process, receiver $\mathrm{R}_{i}$ will construct matrix $\mathbf{D}_{i}$ from the rows of $\mathbf{G}$ that are associated to successfully received packets. The number of rows that sub-matrices $\mathbf{I}_{N \times N}$ and $\mathbf{C}_{\Delta \times K}$ will contribute to $\mathbf{D}_{i}$ depends on the link conditions, which are captured by the packet erasure probability $\varepsilon_{i}$ and the average burst length $L_{i}$. Receiver $\mathrm{R}_{i}$ will recover $\mathbf{u}_{1}, \ldots, \mathbf{u}_{K}$ if and only if $\mathbf{D}_{i}$ contains $K$ linearly independent rows or, equivalently, the rank of $\mathbf{D}_{i}$ is $K$, i.e., $\operatorname{rk}\left(\mathbf{D}_{i}\right)=K$.

The structure of matrix $\mathbf{C}_{\Delta \times K}$ as well as the value of $\Delta$ are determined by the adopted transmission scheme and the content of the feedback that is sent by the main stations, as explained in the remainder of this section.

\section{A. Uncoded Transmission (UT)}

In the context of this paper, uncoded transmission implies that data packets are not linearly combined at layers higher than the physical layer. We assume that the effect of forward error correction at the physical layer can be encapsulated in the packet erasure probability and the average burst length of the 'extended channel' that is composed of the channel encoder at the satellite, the wireless channel and the channel decoder at a receiver.

In uncoded transmission, each main station reports to the satellite the indices of the data packets that have not been delivered successfully during the broadcast stage. The satellite collects the feedback messages and retransmits the union of all requested data packets during the retransmission stage. For example, if data packet $\mathbf{u}_{k}$ is retransmitted at time step $\delta$, then row $\delta$ of matrix $\mathbf{C}_{\Delta \times K}$ will contain value 1 in position $k$, while all other 
entries of row $\delta$ will be set to zero, where $1 \leq k \leq K$ and $1 \leq \delta \leq \Delta$. This process is repeated until all main stations recover the $K$ data packets $(\Delta<N-K)$ or the satellite transmits the maximum allowable number of packets $(\Delta=N-K)$.

Example 1. Let $\mathbf{u}_{1}, \ldots, \mathbf{u}_{4}$ be the data packets transmitted by the satellite to two main stations, $\mathrm{R}_{1}$ and $\mathrm{R}_{2}$, during the broadcast stage. Assume that $\mathrm{R}_{1}$ requests the retransmission of $\mathbf{u}_{2}$, while $\mathrm{R}_{2}$ needs $\mathbf{u}_{1}$ and $\mathbf{u}_{4}$. The satellite sends $\mathbf{x}_{5}=\mathbf{u}_{1}, \mathbf{x}_{6}=\mathbf{u}_{2}$ and $\mathbf{x}_{7}=\mathbf{u}_{4}$. If $\mathbf{x}_{5}$ is not received by $\mathrm{R}_{2}$, the satellite sends $\mathrm{x}_{8}=\mathbf{u}_{1}$. The retransmission stage can thus be summarized by

$$
\mathbf{C}_{4 \times 4}=\left[\begin{array}{llll}
1 & 0 & 0 & 0 \\
0 & 1 & 0 & 0 \\
0 & 0 & 0 & 1 \\
1 & 0 & 0 & 0
\end{array}\right] .
$$

\section{B. Index Coding (IC)}

At the end of the broadcast stage, main stations send feedback messages to the satellite to request missing data packets, as in uncoded transmission. When index coding [6] is employed, the satellite uses the feedback messages to build a 'received' set and a 'requested' set for each main station; the former set contains data packets that have been successfully delivered to the main station and the latter set consists of data packets that are needed by the main station. Given that all main stations aim to recover the flight data, the union of the 'received' and 'requested' sets of each main station contains all of the $K$ data packets. The encoding process takes into account the sets of all main stations to identify data packets that will be added and generate coded packets. Operations on data packets are over the finite field $\mathbb{F}_{2}$, that is, addition is equivalent to the bit-by-bit XOR operation. Main stations can perform XOR between received coded packets and data packets in their 'received' sets in order to derive data packets that are members of the 'requested' sets. The objective of index coding is to generate and transmit the minimum number of coded packets that will enable all main stations to derive their requested data packets.

The Least Difference Greedy (LDG) clique-cover algorithm, which was proposed in [6] for the construction of coded packets, has been adapted to the system model that is considered in this paper. Coded packets are retransmitted if they are needed but not received by one or more main stations, as long as the maximum allowable number of packet transmissions is not exceeded.

Example 2. As in Example 1, $\mathrm{R}_{1}$ and $\mathrm{R}_{2}$ request data packets $\mathbf{u}_{2}$ and $\left\{\mathbf{u}_{1}, \mathbf{u}_{4}\right\}$, respectively. Index coding at the satellite generates $\mathbf{x}_{5}=\mathbf{u}_{1}+\mathbf{u}_{2}$ and $\mathbf{x}_{6}=\mathbf{u}_{4}$. If $\mathbf{x}_{5}$ is not delivered to $R_{2}$, the satellite resends $\mathbf{x}_{7}=\mathbf{u}_{1}+\mathbf{u}_{2}$. Recall that $\mathrm{R}_{1}$ received $\mathbf{u}_{1}$ in the broadcast stage and can perform $X O R$ between $\mathbf{x}_{5}$ and $\mathbf{u}_{1}$ to derive $\mathbf{u}_{2}$, i.e., $\mathbf{u}_{1}+\mathbf{x}_{5}=\mathbf{u}_{2}$. Similarly, $\mathrm{R}_{2}$ obtains $\mathbf{u}_{2}+\mathbf{x}_{7}=\mathbf{u}_{1}$. The retransmission stage is described by

$$
\mathbf{C}_{3 \times 4}=\left[\begin{array}{llll}
1 & 1 & 0 & 0 \\
0 & 0 & 0 & 1 \\
1 & 1 & 0 & 0
\end{array}\right] .
$$

\section{Random Linear Network Coding (RLNC)}

Let $\mathcal{M}$ be the set of indices of receivers that are main stations not in possession of all of the $K$ data packets. Also, let $|\mathcal{M}| \leq S$ be the cardinality of $\mathcal{M}$. When random linear network coding (RLNC) [7] is used, main stations report to the satellite the number - but not the indices - of data packets that they have collected. This is equivalent to reporting the rank of each matrix $\mathbf{D}_{m}$ for $m \in \mathcal{M}$. The satellite determines and transmits the maximum number of required coded packets based on $K-\min \left\{\operatorname{rk}\left(\mathbf{D}_{1}\right), \ldots, \operatorname{rk}\left(\mathbf{D}_{|\mathcal{M}|}\right)\right\}$. Coded packets are random linear combinations of data packets. In other words, the elements of $\mathbf{C}_{\Delta \times K}$ are selected uniformly at random from $\mathbb{F}_{q}$, where $q$ is a prime power. For a fair comparison between RLNC and index coding, the encoding operations are performed over $\mathbb{F}_{2}$ in this paper. After the coded packets have been transmitted, main stations update their matrices $\mathbf{D}_{1}, \ldots, \mathbf{D}_{|\mathcal{M}|}$, recalculate their ranks and inform the satellite. Additional coded packets are randomly generated and transmitted until all main stations recover the flight data or the maximum allowable number of packet transmissions is reached.

Example 3. Similarly to the previous examples, $\mathrm{R}_{1}$ and $\mathrm{R}_{2}$ have collected $\left\{\mathbf{u}_{1}, \mathbf{u}_{3}, \mathbf{u}_{4}\right\}$ and $\left\{\mathbf{u}_{2}, \mathbf{u}_{3}\right\}$, respectively, hence $\operatorname{rk}\left(\mathbf{D}_{1}\right)=3$ and $\operatorname{rk}\left(\mathbf{D}_{2}\right)=2$. The satellite randomly generates and transmits two coded packets, for example $\mathbf{x}_{5}=\mathbf{u}_{1}+\mathbf{u}_{2}+\mathbf{u}_{3}$ and $\mathbf{x}_{6}=\mathbf{u}_{2}+\mathbf{u}_{4}$. If both stations receive any of the two coded packets, then $\operatorname{rk}\left(\mathbf{D}_{1}\right)=4$ and $\operatorname{rk}\left(\mathbf{D}_{2}\right)=3$. The satellite subsequently transmits one more randomly generated coded packet, for example $\mathbf{x}_{7}=\mathbf{u}_{1}+\mathbf{u}_{4}$. The matrix that describes the retransmission stage can be expressed as

$$
\mathbf{C}_{3 \times 4}=\left[\begin{array}{llll}
1 & 1 & 1 & 0 \\
0 & 1 & 0 & 1 \\
1 & 0 & 0 & 1
\end{array}\right] .
$$

\section{RLNC with Partial Packet Recovery (RLNC-PPR)}

Let $\mathcal{S}_{i}$ be the set of packets that have been correctly recovered by the physical layer of receiver $\mathrm{R}_{i}$ and forwarded to higher layers. On the other hand, let set $\mathcal{F}_{i}$ consist of packets that contain irrecoverable bit errors. Based on those definitions, $\left|\mathcal{S}_{i}\right|+\left|\mathcal{F}_{i}\right|=K+\Delta$. The size of $\mathcal{F}_{i}$ is proportional to the channel parameters $\varepsilon_{i}$ and $L_{i}$. If matrix $\mathbf{X}$ contains the $K+\Delta$ packets transmitted by the satellite, as shown in (1), let $\mathbf{Y}_{i}$ be the matrix composed of the packets in $\mathcal{S}_{i} \cup \mathcal{F}_{i}$ as rows. The relationship $\mathbf{Y}_{i}=\mathbf{X}+\mathbf{E}_{i}$ holds, where $\mathbf{E}_{i}$ is referred to as the error matrix. Nonzero elements of $\mathbf{E}_{i}$ identify the bits in $\mathbf{X}_{i}$ that have been flipped by the channel. 
In RLNC, the packets in $\mathcal{F}_{i}$ are discarded. Matrix $\mathbf{D}_{i}$ is built from the rows of $\mathbf{G}$ in (1) that are associated to the packets in $\mathcal{S}_{i}$. In essence, the packets in $\mathcal{S}_{i}$ and matrix $\mathbf{D}_{i}$ compose a linear system of equations over $\mathbb{F}_{2}$ that can be solved if $K$ of the equations are linearly independent, i.e., $\operatorname{rk}\left(\mathbf{D}_{i}\right)=K$, as previously explained.

RLNC with partial packet recovery (RLNC-PPR) [8] uses principles of compressive sensing to repair and utilize some of the packets in $\mathcal{F}_{i}$. According to RLNCPPR, receivers have knowledge of matrix $\mathbf{G}$. This can be achieved if each row of $\mathbf{C}_{\Delta \times K}$ is created by a pseudorandom number generator. Knowledge of the generator's seed and the index of a packet, whether the packet has been received in error or not, can be used by a receiver to reconstruct the corresponding row of $\mathbf{C}_{\Delta \times K}$. Appending $\mathbf{C}_{\Delta \times K}$ to $\mathbf{I}_{K \times K}$ produces $\mathbf{G}$. Based on $\mathbf{G}$, a new matrix $\mathbf{H}$ can be designed, such that $\mathbf{H G}=\mathbf{0}$. If $\mathcal{S}_{i}$ does not hold $K$ linearly independent packets, receiver $\mathrm{R}_{i}$ builds matrix $\mathbf{Y}_{i}$ from the packets in $\mathcal{S}_{i} \cup \mathcal{F}_{i}$. Multiplication of $\mathbf{H}$ by $\mathbf{Y}_{i}$ gives:

$$
\begin{aligned}
\mathbf{H Y}_{i}=\mathbf{A} & \Leftrightarrow \mathbf{H}\left(\mathbf{X}+\mathbf{E}_{i}\right)=\mathbf{A} \\
& \Leftrightarrow \mathbf{H}\left(\mathbf{G} \mathbf{U}+\mathbf{E}_{i}\right)=\mathbf{A} \\
& \Leftrightarrow \mathbf{H} \mathbf{E}_{i}=\mathbf{A} .
\end{aligned}
$$

Matrices $\mathbf{H}$ and $\mathbf{Y}_{i}$ are available to receiver $\mathrm{R}_{i}$, and matrix $\mathbf{A}$ can be obtained from the product $\mathbf{H} \mathbf{Y}_{i}$. Thus, the objective of $\mathrm{R}_{i}$ is to determine the best estimate of $\mathbf{E}_{i}$, denoted by $\hat{\mathbf{E}}_{i}$, that satisfies equation (2). This is a common problem in compressive sensing that can be solved by conventional techniques, such as per-column basic pursuit [8], [13]. Adding $\hat{\mathbf{E}}_{i}$ to $\mathbf{Y}_{i}$ produces an estimate of $\mathbf{X}$, that is, $\hat{\mathbf{X}}=\mathbf{Y}_{i}+\hat{\mathbf{E}}_{i}$. Rows of $\hat{\mathbf{X}}$ that correspond to successfully repaired packets are added to set $\mathcal{S}_{i}$, matrix $\mathbf{D}_{i}$ is updated and its rank is recalculated. If the rank of $\mathbf{D}_{i}$ is still less than $K$, additional packet transmissions are requested, as in RLNC.

\section{Results And Discussion}

The four forwarding schemes (UT, IC, RLNC and RLNC-PPR) in the three system configurations under investigation (independent main stations, connected main stations and a single main station connected to auxiliary stations) are compared in this section. Their performance has been measured in terms of the number of packets that need to be transmitted by the satellite, under certain channel conditions, for all main stations to decode the flight data with a target probability. For instance, if $Z$ is a random variable that gives the number of packets transmitted by the satellite, then the cumulative distribution function $P(Z \leq K+\Delta)$ represents the probability that all main stations will decode the $K$ data packets after $K+\Delta$ packet transmissions. Henceforth, we shall refer to $P(Z \leq K+\Delta)$ as the decoding probability.

Simulations considered systems with $S$ receivers, for $S \in\{1,2,3\}$. In all system configurations, receiver $\mathrm{R}_{1}$

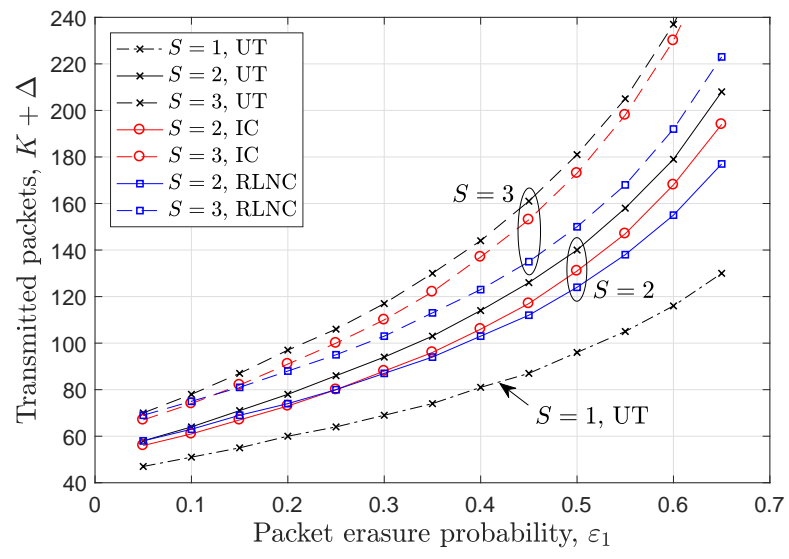

Fig. 3. Number of packets required to be transmitted by the satellite to $S$ independent main stations, for $S=1,2,3$, over channels of different erasure probabilities to achieve a decoding probability of $95 \%$.

is a main station. The packet erasure probability of the channel between the satellite and receiver $R_{1}$ was varied in the range $\varepsilon_{1} \in[0.05 \ldots 0.65]$. The average length of erasure bursts was set equal to $L_{1}=2$ packets. The remaining receivers were assumed to experience worse channel conditions than $\mathrm{R}_{1}$. The channel parameters for $\mathrm{R}_{i}$ are given by $\varepsilon_{i}=\varepsilon_{i-1}+0.05$ and $L_{i}=L_{i-1}+2$, for $1<i \leq S$. Flight information was divided into blocks, each consisting of $K=40$ data packets. The length of a data packet was taken to be 128 bits. The LDG algorithm for IC was implemented in MATLAB. The compressive sensing problem in (2) for RLNC-PPR was solved using the basic pursuit algorithm of SparseLab [14].

Simulation results determined the number of packets that need to be transmitted by the satellite, on average, to enable all main stations to decode all data packets with probability $95 \%$ for different values of $\varepsilon_{1}$. Fig. 3 shows the impact of increasing the number of independent main stations on packet transmissions. Additional main stations provide redundancy and allow parallel processing of recovered flight data at the cost of a higher number of packet transmissions. For $S>1$, UT is the least efficient scheme for any value of $\varepsilon_{1}$. IC offers a negligible gain at very low packet erasure probabilities but its performance converges to that of UT as the value of $\varepsilon_{1}$ increases. For a fixed number of transmitted packets and medium to high values of $\varepsilon_{1}$, RLNC can support a decoding probability of $95 \%$ at worse channel conditions than UT and IC.

The performance curves of UT, IC and RLNC, when the main stations are connected, are depicted in Fig. 4. Note that the performance of UT for $S=1$, which is clearly identified on Figs. 3-5, is the same for all system configurations and is used as a benchmark. For high values of $\varepsilon_{1}$, a larger number of main stations causes the transmission of an overwhelming number of requests for specific coded packets and reduces the performance of a system based on UT or IC. The situation is reversed as the channel conditions improve because reception of 


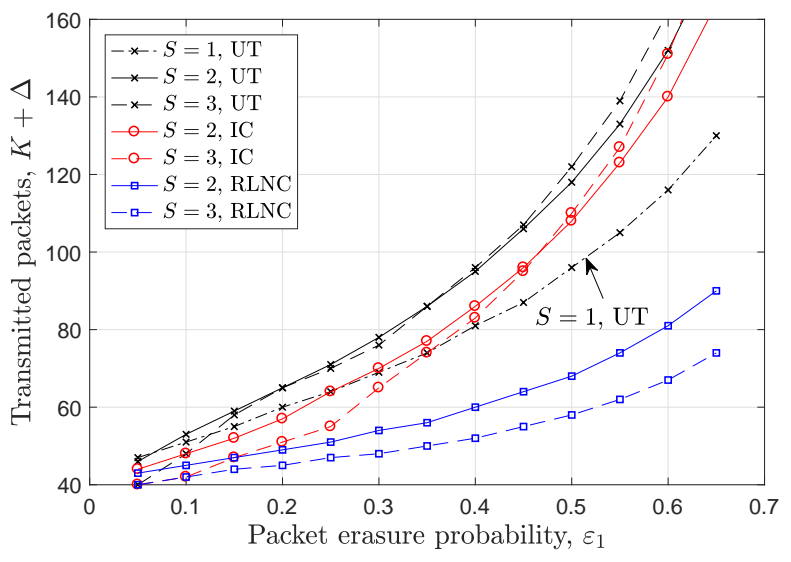

Fig. 4. Number of packets required to be transmitted by the satellite to $S$ connected main stations, for $S=1,2,3$, over channels of different erasure probabilities to achieve a decoding probability of $95 \%$.

each packet by at least one main station is more likely. Hence, the union of all packets received by all main stations can lead to the recovery of the flight data with a higher probability. In contrast to IC, which transmits copies of undelivered coded packets, RLNC transmits different randomly-generated coded packets. As a result, linear independence between packets in the union of all received packets is more probable, and the likelihood of recovering the flight data is higher than in IC.

Fig. 5 shows the performance gain from having multiple receivers but only one main station among them. In all cases, an increase in the number of auxiliary stations improves the overall performance. The notable advantage of combining PPR with RLNC is also illustrated. For example, assume that $S=3$ and $\varepsilon_{1}=0.6$. As seen in Fig. 5, RLNC cannot guarantee a decoding probability of $95 \%$ if fewer than 67 packets are transmitted. If RLNC decoding fails to recover the flight data, RLNC-PPR decoding can be performed on the same received packets and attain a decoding probability of $95 \%$, provided that at least 44 packets have been broadcast. Therefore, the main station could perform RLNC decoding in real time and RLNC-PPR decoding offline in order to increase the amount of recovered flight data.

\section{CONCLUSions}

This paper considered a satellite that stores and transmits vital flight information to authorized recipients over independent links that have been modeled as Gilbert channels. The transmission of packets containing flight data is a two-stage process; the broadcast stage is followed by the retransmission stage. The latter stage is controlled by feedback messages sent by recipients to the satellite. Packet transmissions can be reduced or resilience to packet erasures can be strengthened if (i) the satellite uses RLNC in the retransmission stage to encode data packets, and (ii) multiple recipients collect coded packets, provided that only one of them sends feedback

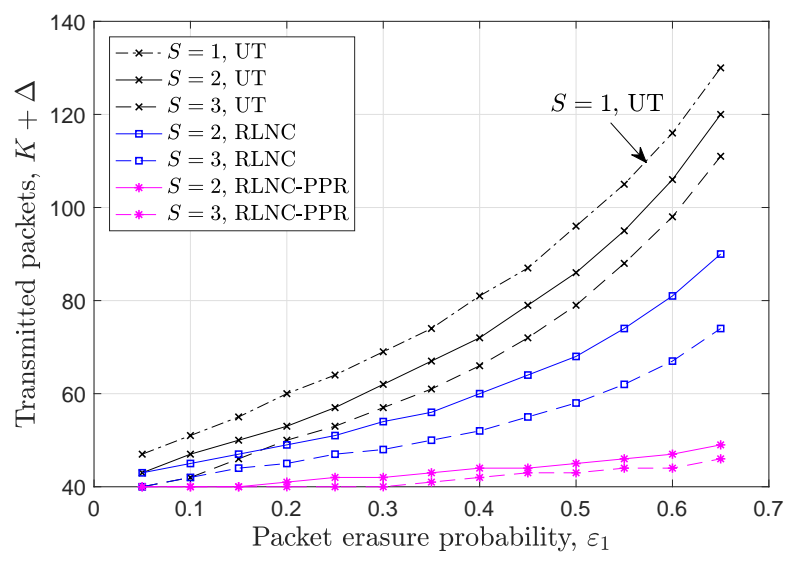

Fig. 5. Number of packets required to be transmitted by the satellite to a single main station and $(S-1)$ auxiliary stations, for $S=1,2,3$, in order to achieve a decoding probability of $95 \%$.

during the retransmission stage and gathers packets from other recipients at the end of the two-stage process. If RLNC decoding cannot fully reconstruct the flight data due to poor channel conditions or a short retransmission stage, PPR can be included in the RLNC decoder to significantly enhance its decoding capability.

\section{REFERENCES}

[1] Y. Yu, "The aftermath of the missing flight MH370: What can engineers do?" Proc. IEEE, vol. 103, no. 11, pp. 1948-1951, Nov. 2015.

[2] "Annex 6: Operation of aircraft," International Civil Aviation Organization, 2016, Amendments 39 and 40.

[3] "SwiftBroadband-safety: The future of aircraft communications," Inmarsat, Nov. 2016.

[4] "SwiftBroadband and IP data connections," Inmarsat, Jan. 2008.

[5] "Inmarsat SB-Safety connected flight deck enters service evaluation on United Airlines," Inmarsat Press Release, Nov. 2017. [Online]. Available: http://bit.ly/2FUqYXJ

[6] Y. Birk and T. Kol, "Coding on demand by an informed source (ISCOD) for efficient broadcast of different supplemental data to caching clients," IEEE Trans. Inf. Theory, vol. 52, no. 6, pp. 2825-2830, Jun. 2006.

[7] T. Ho, M. Médard, R. Koetter, D. R. Karger, M. Effros, J. Shi, and B. Leong, "A random linear network coding approach to multicast," IEEE Trans. Inf. Theory, vol. 52, no. 10, pp. 44134430 , Oct. 2006.

[8] M. S. Mohammadi, Q. Zhang, and E. Dutkiewicz, "Reading damaged scripts: Partial packet recovery based on compressive sensing for efficient random linear coded transmission," IEEE Trans. Commun., vol. 64, no. 8, pp. 3296-3310, Aug. 2016.

[9] E. N. Gilbert, "Capacity of a burst-noise channel," Bell Syst. Tech. J., vol. 39, no. 5, pp. 1253-1266, Sep. 1960.

[10] R. Alegre-Godoy and M. A. Vázquez-Castro, "Spatial diversity with network coding for ON/OFF satellite channels," IEEE Commun. Lett., vol. 17, no. 8, pp. 1612-1615, Aug. 2013.

[11] J. Cloud and M. Médard, "Network coding over SATCOM: Lessons learned," in Proc. Int. Conf. on Wireless and Satellite Systems (WiSATS), Bradford, UK, Jul. 2015, pp. 272-285.

[12] T. Rancurel, D. Roviras, and F. Castanié, "Expression of the capacity for the Gilbert channel in presence of interleaving," in Proc. IEEE Int. Conf. on Acoustics, Speech and Signal Processing (ICASSP), Salt Lake City, USA, May 2001, pp. 2533-2536.

[13] D. L. Donoho, "Compressed sensing," IEEE Trans. Inf. Theory, vol. 52, no. 4, pp. 1289-1306, Apr. 2006.

[14] SparseLab: Seeking sparse solutions to linear systems of equations. Stanford University. Accessed on March 21, 2018. [Online]. Available: https://sparselab.stanford.edu 